\title{
TOXICIDAD DE SCHINUS MOLLE L. (ANACARDIACEAE) A CUATRO CONTROLADORES BIOLÓGICOS DE PLAGAS AGRÍCOLAS EN EL PERÚ
}

\author{
José IANNACONE ${ }^{1,2}$, Lorena ALVARIÑO 2 \\ ${ }^{1}$ Laboratorio de Invertebrados - Museo de Historia Natural. Facultad de Ciencias Biológicas. Univer- \\ sidad Ricardo Palma. Av. Benavides 5440, Lima 33, PERÚ. \\ ${ }^{2}$ Laboratorio de Ecofisiología Animal. Facultad de Ciencias Naturales y Matemática. Universidad \\ Nacional Federico Villarreal, Av. Río de Chepén, s/n. Urb. Villa Hermosa, El Agustino, Lima, PERÚ.
}

Iannacone, J. \& L. Alvariño. 2010. Toxicidad de Schinus molle L. (Anacardiaceae) a cuatro controladores biológicos de plagas agrícolas en el Perú. Acta Zool. Mex. (n. s.), 26(3): 603-615.

RESUMEN. Schinus molle L. (Anacardiaceae) "molle" es una planta de importancia etnobotánica originaria de la zona altoandina y usada artesanalmente como agente insecticida para el control de plagas agrícolas en el Perú. Se evaluó el efecto ecotoxicológico de extractos acuosos de molle sobre cuatro organismos no blanco: Ceraeochrysa cincta (Schneider) (Neuroptera: Chrysopidae) en huevos y larvas de primer estadío, Chrysoperla asoralis (Bank) (Neuroptera: Chrysopidae) en huevos y larvas de primer estadío, Telenomus remus Nixon (Scelionidae: Hymenoptera) en adultos y Orius insidiosus Say (Anthocoridae: Hemiptera) en adultos, bajo condiciones de laboratorio. Se emplearon cinco concentraciones acuosas de hojas de $S$. molle (p/v): $1.5 \%, 2.5 \%, 5 \%, 10 \%$ y $20 \%$. Los parámetros de toxicidad aguda empleados fueron la concentración letal $\left(\mathrm{CL}_{50}\right)$ y la efectiva media $\left(\mathrm{CE}_{50}\right)$. Se encontró la siguiente secuencia en orden decreciente de toxicidad en términos de $\mathrm{CE}_{50} / \mathrm{CL}_{50}$ : mortalidad larvaria de $C$. cincta $\left(\mathrm{CL}_{50}\right.$ a $\left.48 \mathrm{~h}=3.7 \%\right)>$ no eclosión de huevos de $C$. $\operatorname{cincta}\left(\mathrm{CE}_{50}=5.1 \%\right)>$ adultos de $O$. insidiosus $\left(\mathrm{CL}_{50}\right.$ a $\left.48 \mathrm{~h}=14.2 \%\right)>$ mortalidad larvaria de $C$. asoralis $\left(\mathrm{CL}_{50}\right.$ a $\left.48 \mathrm{~h}=32.2 \%\right)>$ no eclosión de huevos de $C$. asoralis $\left(\mathrm{CE}_{50}=34.3 \%\right)>$ adultos de $T$. remus $\left(\mathrm{CL}_{50}\right.$ a $\left.48 \mathrm{~h}=40.9 \%\right)$. Se analiza la posibilidad de riesgo ecológico del empleo de este insecticida botánico en el agroecosistema peruano. Se incluye una lista de todas las especies eucariotas donde se ha evaluado la toxicidad y repelencia del molle.

Palabras clave: Ceraeochrysa, Chrysoperla, insecticida botánico, Orius, Schinus, Telenomus.

Iannacone, J. \& L. Alvariño. 2010. Toxicity of Schinus molle L. (Anacardiaceae) on four biological control agents of agriculture pest in Peru. Acta Zool. Mex. (n. s.), 26(3): 603-615.

ABSTRACT. Schinus molle L. (Anacardiaceae) "pepper tree" is an ethnobotanical important plant belonging to Andean zone and is used as a botanical insecticidal agent for agricultural pest control. The ecotoxicological effects of aqueous extracts of the pepper tree on the following four non-target organisms were evaluated: Ceraeochrysa cincta (Schneider) (Neuroptera: Chrysopidae) eggs and firstinstar larvae, Chrysoperla asoralis (Bank) (Neuroptera: Chrysopidae) eggs and first-instar larvae, Telenomus remus Nixon (Scelionidae: Hymenoptera) adults, and Orius insidiosus Say (Anthocoridae: Hemiptera) adults. Five concentrations with increasing amounts of an aqueous extract from $S$. molle

Recibido: 26/01/2010; aceptado: 22/06/2010. 
leaves (w/v) were employed: $1.5 \%, 2.5 \%, 5 \%, 10 \%$ and $20 \%$. The parameters of acute toxicity employed were the lethal concentration $\left(\mathrm{LC}_{50}\right)$ and the effective concentration $\left(\mathrm{EC}_{50}\right)$. The following sequence in decresing order of ecotoxicity in terms of $\mathrm{EC}_{50} / \mathrm{LC}_{50}$ were found: C. cincta larval mortality $\left(\mathrm{LC}_{50}\right.$ at $\left.48 \mathrm{~h}=3.7 \%\right)>$ not hatched $C$. cincta eggs $\left(\mathrm{EC}_{50}=5.1 \%\right)>O$. insidiosus adults $\left(\mathrm{LC}_{50}\right.$ at $48 \mathrm{~h}$ $=14.2 \%)>C$. asoralis larval mortality $\left(\mathrm{LC}_{50}\right.$ at $\left.48 \mathrm{~h}=32.2 \%\right)>$ not hatched $C$. asoralis eggs $\left(\mathrm{EC}_{50}=\right.$ $34.3 \%)>T$. remus adults $\left(\mathrm{LC}_{50}\right.$ a $\left.48 \mathrm{~h}=40.9 \%\right)$. Potential ecological risks of employing this botanical insecticide in Peruvian agroecosystems are discussed. A list of all eukaryotic species with toxicity and repellence of the pepper tree evaluated is included.

Key words: Ceraeochrysa, Chrysoperla, Orius, Schinus, Telenomus.

\section{INTRODUCCIÓN}

Schinus molle L. (Anacardiaceae) "molle" o "árbol del Perú" es una planta con propiedades aromatizantes, se utiliza como chicle, colorante, combustible, comestible, condimento, cosmético, curtiente, forrajero, melífera, medicinal e insecticida. S. molle mejora la fertilidad y erosión del suelo, es barrera contra vientos, ornamental y actúa como sombra y refugio para la vida silvestre (Conabio, 2009). Esta planta de importancia etnobotánica es originaria de la zona altoandina en Sudamérica y en el Perú, es usada artesanalmente como agente insecticida para el control de plagas agrícolas. Es importante determinar el nivel de riesgo ambiental de los insecticidas como el molle sobre los controladores biológicos usando bioensayos ecotoxicológicos (Wu et al. 2004a, b). Con este objetivo en mira fueron seleccionadas cuatro especies de controladores biológicos para realizar ensayos toxicológicos:

1) Orius insidiosus (Say, 1832) (Hemiptera: Anthocoridae), un depredador importante de diferentes plagas económicas en algodón, en invernadero y en plantas ornamentales. Se ha evaluado la toxicidad de varios plaguicidas sobre esta chinche benéfica (Ludwig \& Oetting 2001; Carvalho et al. 2002; Studebaker \& Kring 2003).

2) Telenomus remus Nixon, 1937 (Hymenoptera: Scelionidae) es un parasitoide de huevos de lepidópteros plaga de Asia y de las Américas. Su alta tasa reproductiva, y su fácil y económico cultivo masivo hace de esta microavispa un buen agente para el control biológico de lepidópteros plaga, particularmente del género Spodoptera (Noctuidae) aunque puede atacar hasta 30 especies plagas diferentes (Morales et al. 2000, 2001; Gazit et al. 2002; Murthy et al. 2004; Bueno et al. 2008). Por lo que de este parasitoide se conoce bien su biología, ecología y su uso en el manejo de plagas (Cave 2000); así como el efecto de insecticidas sintéticos en la actividad de esta microavispita (Waddill 1978).

3) Ceraeochrysa cincta Schneider, 1851 (Neuroptera: Chrysopidae) es una especie ampliamente distribuida desde Florida, Estados Unidos de América (EUA), hasta Argentina, incluyendo las Islas Galápagos en América del Sur (Tauber \& De León 2001; Ramírez et al. 2007). Se encuentra asociada a diversos artrópodos plaga en diferentes sistemas agrícolas, con gran potencial para la cría masiva y utilización 
en programas de control biológico (Nuñez 1988; López-Arroyo et al. 1999a, b; Tauber \& De León 2001). Se le ha evaluado depredando ácaros, Bemisia tabaci (Gennadius, 1889) (Homoptera: Aleyrodidae), cóccidos y pulgones, entre otros artrópodos plaga (Ramírez et al. 2007); así como su historia de vida, nutrición y su sensibilidad a entomopatógenos (López-Arroyo et al. 1999b; Cardoso et al. 2004; Bortoli et al. 2005).

4) Chrysoperla asoralis (Banks, 1915) (Neuroptera: Chrysopidae) es una especie depredadora perteneciente de la región Neotropical y es de importancia en el cultivo de espárrago (Gonzáles \& Reguillón 2002). En Perú, Colombia, Norteamérica, Europa y México ha sido utilizada con mucho éxito para el control de plagas en hortalizas y frutales tanto en campo abierto como en invernadero. Las condiciones climáticas del Perú, especialmente la costa (donde hay ausencia de lluvias y temperaturas favorables para las crisopas durante todo el año), hacen que este depredador tenga especial éxito.

Por otro lado, se han desarrollado diferentes protocolos de bioensayos para determinar el efecto de plaguicidas sobre la fauna benéfica (Calow 1993; Iannacone et al. 2000; Iannacone \& Alvariño 2005). El parámetro de toxicidad aguda más comúnmente empleado es la concentración letal media $\left(\mathrm{CL}_{50}\right)$ (en $\mathrm{mg}$ o $\mu \mathrm{g} \mathrm{L} \mathrm{L}^{-1}$ ) o la dosis letal media $\left(\mathrm{DL}_{50}\right)\left(\mathrm{mg} \mathrm{o}_{\mathrm{g} \mathrm{kg}} \mathrm{kg}^{-1}\right)$ (Throne et al. 1995). Los ensayos tendientes a evaluar la toxicidad crónica son también ampliamente utilizados (Calow 1993). De esta forma, el objetivo de este trabajo fue evaluar el efecto ecotoxicológico de extractos acuosos de molle sobre cuatro organismos no blanco: Ceraeochrysa cincta (Schneider) (Neuroptera: Chrysopidae) en huevos y larvas de primer estadío, Chrysoperla asoralis (Bank) (Neuroptera: Chrysopidae) en huevos y larvas de primer estadío, Telenomus remus Nixon (Scelionidae: Hymenoptera) en adultos y Orius insidiosus Say (Anthocoridae: Hemiptera) en adultos, bajo condiciones de laboratorio y a partir de estos resultados evaluar el riesgo ambiental de este insecticida artesanal en el ambiente terrestre.

\section{MATERIALES Y METODOS}

Diseño experimental . Las pruebas de toxicidad aguda para $O$. insidiosus, $T$. remus, C. cincta y $C$. asoralis, usadas para $S$. molle incluyeron cinco concentraciones más el control: $1.5 \%, 2.5 \%, 5 \%, 10 \%$ y $20 \%$, cada una con cuatro repeticiones, en un diseño en bloque completamente aleatorio (DBCA). C. cincta y C. asoralis tuvieron también pruebas con la eclosión de los huevos, a las mismas concentraciones que las pruebas agudas. Los criterios de selección de las concentraciones empleadas fueron de acuerdo a Iannacone et al. (2008).

Material biológico. Schinus molle. La especie fue seleccionada debido a sus antecedentes para controlar plagas (Chirino et al. 2001; Pérez-Pacheco et al. 2004). 
Las hojas de molle se emplearon para la preparación de los extractos acuosos crudos. Los especímenes botánicos se obtuvieron de jardines adyacentes a la Facultad de Ciencias Biológicas de la Universidad Ricardo Palma (URP), en octubre de 2007. La recolección del material vegetal se realizó en la etapa de floración. Las hojas fueron secadas en estufa a $40{ }^{\circ} \mathrm{C}$ durante $48 \mathrm{~h}$, hasta obtener un peso seco constante, posteriormente fueron trituradas en un mortero. Se prepararon extractos acuosos crudos al 20\%, en una proporción de $20 \mathrm{~g}$ por $100 \mathrm{~mL}$ de agua destilada, se mantuvieron en maceración constante por 48 h para la extracción de los compuestos hidrosolubles. Posteriormente se filtraron a través de un papel filtro (Whatman ${ }^{\circledR}$ $\mathrm{N}^{\circ} 1$ ). Finalmente se prepararon las concentraciones al 1.5\%, 2.5\%, 5\%, $10 \%$ y $20 \%$ más el control (Iannacone \& Lamas 2003a).

Orius insidiosus y Telenomus remus: la chinche pirata $O$. insidiosus, y $T$. remus fueron obtenidos de colonias mantenidas por el Programa Nacional de Control Biológico-Servicio Nacional de Sanidad Agraria (PNCB-SENASA), Lima, Perú. $O$. insidiosus fue criada en el laboratorio en esquejes de camote con huevos de Sitotroga cerealella (Olivier, 1819). T. remus fue mantenida en el laboratorio sobre larvas de Spodoptera eridania (Cramer, 1782). Las condiciones de temperatura para las crías de ambas especies fueron de $22 \pm 3{ }^{\circ} \mathrm{C}$, humedad relativa de $80 \pm 10 \%$ y a $12 \mathrm{~h}$ de fotoperíodo (Cano 2001). Los ensayos de contacto residual se llevaron a cabo para los adultos de $O$. insidiosus y $T$. remus, y se desarrollaron de la manera siguiente: los envases de plástico de $8 \mathrm{~mL}$ fueron cubiertos con una tapa de algodón, con una pipeta automática (Thermo Electrón Labsystems®, Finlandia) se les agregó $500 \mu \mathrm{L}$ de cada una de las concentraciones acuosas, y se esparcieron homogéneamente con un hisopo sobre la superficie interna. Posteriormente se permitió el secado de los viales a temperatura ambiente durante $2 \mathrm{~h}$ o alternativamente a una temperatura de $35{ }^{\circ} \mathrm{C}$ en una estufa durante $1 \mathrm{~h}$, con sus respectivos tapones o torundas de algodón. Los experimentos se realizaron con cohortes de adultos con menos de $24 \mathrm{~h}$ de emergidos y no alimentados antes del bioensayo. Se emplearon individuos macho y hembra al azar, tomados de los frascos de emergencia de adultos de $O$. insidiosus y $T$. remus. Para cada una de las pruebas se utilizaron 240 individuos, con 40 organismos por cada una de las seis concentraciones y 10 por repetición. Se consideraron muertos los organismos que se encontraban con las patas dirigidas hacia arriba. El tratamiento control consistió en agua destilada. Se usaron cuatro repeticiones por tratamiento. Posteriormente, se condujeron ensayos de toxicidad aguda estáticos y de residuos en condiciones de oscuridad. Los envases se mantuvieron en condiciones de cría y oscuridad y se observó la mortalidad acumulada a diferentes h de exposición, hasta $48 \mathrm{~h}$. Las lecturas se continuaron siempre y cuando la mortalidad en el control no fuera mayor al $10 \%$ y éstas se corrigieron con la fórmula de Abbott (Abbott 1925). Este bioensayo siguió lo señalado por Iannacone et al. (2008). 
Ceraeochrysa cincta y Chrysoperla asoralis: Las condiciones de cría para los crisópidos para la obtención de huevos y larvas fueron de acuerdo a lo descrito por Iannacone \& Lamas (2002). Para los bioensayos, se emplearon huevos de menos de $48 \mathrm{~h}$ y larvas de menos de $24 \mathrm{~h}$. Para el ensayo de eclosión de huevos, éstos fueron incubados individualmente en pequeños envases de plástico de $8 \mathrm{~mL}$ de capacidad. Para el ensayo de mortalidad larvaria, éstas fueron criadas individualmente en envases de plástico de $12 \mathrm{~mL}$ de capacidad y alimentadas ad libitum con huevos de $S$. cerealella, pegados en cartulinas de $5 \times 5 \mathrm{~mm}$. Las larvas fueron criadas hasta el primer estadio de desarrollo y se emplearon cohortes de especímenes entre 24 a $48 \mathrm{~h}$ de edad. Se escogió este estadio debido a que en bioensayos ecotoxicológicos preliminares se observó que el primer estadío era el más sensible.

Ecotoxicidad por inmersión (ensayo de eclosión de huevos). Los huevos de $C$. cincta y $C$. asoralis se expusieron por inmersión durante $5 \mathrm{~s}$ a las diluciones seleccionadas de $S$. molle y en agua destilada (grupo control). Posteriormente los huevos fueron colocados en papel Tissue ${ }^{\circledR}$ por 10 min para absorber los restos de soluciones acuosas y permitir el secado a temperatura ambiente. Se trataron 20 huevos por cada concentración (5 especímenes por cada una de las cuatro repeticiones). Los huevos fueron individualizados en envases de plástico de $8 \mathrm{~mL}$ de capacidad. Después de las aplicaciones tópicas, los envases de plástico se mantuvieron en oscuridad bajo condiciones de cría, realizándose las lecturas hasta la eclosión de los huevos ( 120 h).

Ecotoxicidad por contacto-residual (ensayo de mortalidad larvaria). Estos ensayos se llevaron a cabo para las larvas de primer estadio de menos de $24 \mathrm{~h}$. El $S$. molle disuelto en agua destilada se aplicó en envases de plástico $(500 \mu \mathrm{L}$ por cada envase de plástico de $12 \mathrm{~mL}$ de capacidad). Con la ayuda de un hisopo de base de madera, en cada envase de plástico se esparció homogéneamente en sus paredes y base, los $\mu \mathrm{L}$ determinados de la sustancia química colocada en su interior; posteriormente se permitió el secado de los envases a temperatura ambiente durante $2 \mathrm{~h}$. En el interior de cada uno de los envases secos, se depositó una larva de primer estadío. Se consideró un total de 40 larvas por repetición. Los envases de plástico se mantuvieron en condiciones de cría y oscuridad; se observó la mortalidad acumulada a 24 y 48 h de exposición. Se consideraron muertos los individuos de $C$. cincta y $C$. asoralis que no realizaron ningún movimiento coordinado en observación de $15 \mathrm{~s}$ al microscopio estereoscopio a $10 \mathrm{x}$ de aumento, con la ayuda de un alfiler entomológico. Las pruebas de sensibilidad se realizaron bajo condiciones de oscuridad, para evitar el efecto de fotolísis (Cardoso et al. 2004). 
Análisis estadístico de datos. Se siguió la clasificación de la IOBC/WPRS (Sterk et al. 1999) para la catalogación toxicológica del extracto de molle en cada uno de los controladores biológicos con base en las pruebas de laboratorio de toxicidad por contacto. En todos los casos, la eficacia de los tratamientos y las repeticiones se evaluaron a través de un análisis de varianza de dos vías con prueba complementaria de significancia de Tukey $(\alpha=0.05)$. Los datos fueron previamente normalizados mediante la transformación raíz cuadrada del arcoseno. Las $\mathrm{CL}_{50}$ o $\mathrm{CE}_{50}$ se calcularon usando el programa computarizado Probit versión 1.5 (Weber 1993). El modelo de regresión fue verificado usando el estadístico $X^{2}$. Se empleó el paquete estadístico SPSS, versión 16 para Windows XP (2001) para el cálculo de los estadísticos descriptivos e inferenciales a un nivel de significancia de $\mathrm{p} \leq 0.05$.

\section{RESULTADOS}

Al evaluar el efecto de contacto del extracto acuoso de $S$. molle a las cinco concentraciones entre 1.5 a $20 \%$ sobre los adultos de $O$. insidiosus se observó que a $24 \mathrm{~h}$ de exposición, la $\mathrm{CL}_{50}$ fue $70.6 \%$ (Cuadro 1). Mientras que a 48 h de exposición los adultos de $O$. insidiosus presentaron una $\mathrm{CL}_{50}$ de $14.2 \%$. En el caso de los adultos del microhimenóptero T. remus, el S. molle a las concentraciones entre 1.5 a $20 \%$ del extracto acuoso la $\mathrm{CL}_{50}$ fue $40.9 \%$ a 48 h de exposición (Cuadro 1). S. molle afectó el porcentaje de mortalidad de $O$. insidiosus y de $T$. remus de $1.5 \%$ y $20 \%$, respectivamente.

Cuadro 1. Efecto de Schinus molle en términos de porcentaje de extracto acuoso en el porcentaje de mortalidad de adultos de Orius insidiosus (Anthocoridae) de 24 a 48 h y de Telenomus remus a 48 h.

\begin{tabular}{lccc}
\hline & \multicolumn{2}{c}{ Orius insidiosus } & Telenomus remus \\
Tiempo & $\mathbf{2 4} \mathbf{~ h}$ & $\mathbf{4 8} \mathbf{~ h}$ & $\mathbf{4 8 ~ \mathbf { ~ }}$ \\
\hline Concentración (\% extracto) & & & \\
& & & \\
Control & $0 \mathrm{a}$ & $0 \mathrm{a}$ & $0 \mathrm{a}$ \\
1.5 & $0 \mathrm{a}$ & $17.6 \mathrm{~b}$ & $0 \mathrm{a}$ \\
2.5 & $10 \mathrm{a}$ & $23.5 \mathrm{~b}$ & $0 \mathrm{a}$ \\
5 & $10 \mathrm{a}$ & $29.4 \mathrm{~b}$ & $6 \mathrm{a}$ \\
10 & $20 \mathrm{ab}$ & $41.1 \mathrm{c}$ & $15 \mathrm{ab}$ \\
20 & $25 \mathrm{~b}$ & $58.8 \mathrm{c}$ & $25 \mathrm{~b}$ \\
$\mathrm{CL}_{50}(\%$ extracto) & 70.6 & 14.2 & 40.9 \\
\hline
\end{tabular}

Porcentajes de mortalidad con letras minúsculas iguales en una misma columna indican que son estadísticamente iguales (Prueba aposteriori de Tukey: $\mathrm{p} \searrow 0.05$ ). 
A las 24 h de exposición por contacto, las larvas de primer estadio de $C$. cincta y $C$. asoralis presentaron una $\mathrm{CL}_{50}$ de $18.2 \%$ y $27.3 \%$, respectivamente (Cuadro 2). A 48 h de exposición $C$. asoralis es 8.7 veces menos sensible al $S$. molle que $C$. cincta en relación a la $\mathrm{CL}_{50}$. El porcentaje de eclosión de huevos de $C$. cincta fue mayor al de C. asoralis, en sensibilidad a S. molle en los ensayos de inmersión (Cuadro 2).

Se encontró la siguiente secuencia en orden decreciente de ecotoxicidad en términos de $\mathrm{CE}_{50} / \mathrm{CL}_{50}$ : mortalidad larvaria de $C$. cincta $\left(\mathrm{CL}_{50}\right.$ a $48 \mathrm{~h}=3.7 \%$ ); no eclosión de huevos de $C$. cincta $\left(\mathrm{CE}_{50}=5.1 \%\right)$; adultos de $O$. insidiosus $\left(\mathrm{CL}_{50}\right.$ a 48 $\mathrm{h}=14.2 \%)$; mortalidad larvaria de C. asoralis $\left(\mathrm{CL}_{50}\right.$ a $\left.48 \mathrm{~h}=32.2 \%\right)$; no eclosión de huevos de $C$. asoralis $\left(\mathrm{CE}_{50}=34.3 \%\right)$; adultos de $T$. remus $\left(\mathrm{CL}_{50}\right.$ a $\left.48 \mathrm{~h}=40.9 \%\right)$.

Cuadro 2. Efecto de Schinus molle en términos de porcentaje de extracto acuoso en el porcentaje de mortalidad de la larva de primer estadio y de no eclosión de huevos de Ceraeochrysa cincta y

Chrysoperla asoralis (Chrysopidae) a 24 y 48 h de exposición.

\begin{tabular}{lcccccc}
\hline & \multicolumn{3}{c}{ Ceraeochrysa cincta } & \multicolumn{3}{c}{ Chrysoperla asoralis } \\
Tiempo & $\mathbf{2 4} \mathbf{~ h}$ & $\mathbf{4 8} \mathbf{~ h}$ & $\mathbf{\%} \mathbf{~ d e}$ & $\mathbf{2 4} \mathbf{~ h}$ & $\mathbf{4 8 ~ h}$ & \% de \\
& & & no eclosión & & & no eclosión \\
\hline Concentración (\% extracto) & & & & & & \\
& & & & & & \\
Control & $0 \mathrm{a}$ & $0 \mathrm{a}$ & $0 \mathrm{a}$ & $0 \mathrm{a}$ & $0 \mathrm{a}$ & $0 \mathrm{a}$ \\
1.5 & $0 \mathrm{a}$ & $25 \mathrm{~b}$ & $22.4 \mathrm{~b}$ & $0 \mathrm{a}$ & $6.4 \mathrm{a}$ & $16.4 \mathrm{ab}$ \\
2.5 & $15 \mathrm{a}$ & $45 \mathrm{c}$ & $50.1 \mathrm{c}$ & $0 \mathrm{a}$ & $0 \mathrm{a}$ & $27.6 \mathrm{~b}$ \\
5 & $20 \mathrm{~b}$ & $50 \mathrm{c}$ & $55.6 \mathrm{c}$ & $3.7 \mathrm{a}$ & $6.8 \mathrm{a}$ & $22.1 \mathrm{~b}$ \\
10 & $32.5 \mathrm{bc}$ & $75 \mathrm{~d}$ & $61.2 \mathrm{c}$ & $19.7 \mathrm{~b}$ & $20.1 \mathrm{~b}$ & $27.6 \mathrm{~b}$ \\
20 & $55 \mathrm{c}$ & $90 \mathrm{e}$ & $61.2 \mathrm{c}$ & $35.8 \mathrm{c}$ & $33.4 \mathrm{~b}$ & $49.8 \mathrm{c}$ \\
$\mathrm{CL}(\mathrm{E})_{50}(\%$ extracto) & 18.2 & 3.7 & 5.1 & 27.3 & 32.2 & 34.3 \\
\hline
\end{tabular}

Letras minúsculas iguales en una misma columna indican que los porcentajes de mortalidad son estadísticamente iguales $(\mathrm{p} \geq 0.05)$

\section{DISCUSIÓN}

Las hojas de $S$. molle contiene taninos, alcaloides, flavonoides, saponinas esferoidales, esteroles, terpenos, gomas, resinas y aceites esenciales, éstos últimos incluyen 20 o más compuestos diferentes (Wimalaratne et al. 1996). Los aceites esenciales se presentan en un $2 \%$ en las hojas del $S$. molle y contienen terpenoides, siendo el cis-menth-2-en-1-ol y el trans-piperitol los que han sido involucrados en la actividad insecticida en Musca domestica L. Por otro lado, Ruffinengo et al. (2005) han encontrado que el canfene, mircene, beta-felandrene y alfa-felandrene son los principales compuestos de los aceites esenciales de las hojas de $S$. molle relacionados 
con la actividad de repelencia e insecticida en Varroa destructor Anderson \& Trueman, 2000 (Mesostigmata: Varroidae) y Apis mellifera L. Algunos de estos compuestos pudieran estar presentes en el extracto acuoso que ha sido evaluado en el presente estudio.

El Cuadro 3 muestra los 27 organismos biológicos eucariontes en los que se han realizado bioensayos para detectar toxicidad y repelencia de $S$. molle. De los organismos encontrados en la literatura disponible, 25 fueron insectos (92.5\%), uno fue ácaro (3.7\%), y uno fue crustáceo (3.7\%). Once fueron plagas agrícolas (40.8\%), nueve organismos benéficos $(33.3 \%)$ y siete de importancia en Salud Pública (25.9\%). La actividad antifúngica de $S$. molle ha sido también evaluada en 20 especies diferentes de hongos (Dikshit et al. 1986; Hernández-Albiter et al. 2007).

$\mathrm{Al}$ extraer distintos metabolitos, los extractos botánicos obtenidos con disolventes acuosos u organicos, pueden ocasionar mortalidades variables sobre los insectos evaluados. En el presente estudio sólo se ha ensayado el extracto crudo acuoso de $S$. molle, que estaría incluyendo una mezcla de sustancias activas de las hojas del molle las cuales pueden actuar sinérgica y antagónicamente.

Extractos acuosos al $10 \%$ de molle no causaron efectos significativos en la mortalidad larvaria y de la pupa de Chrysoperla externa Hagen (Iannacone \& Lamas 2003a). En el presente estudio, en los chrisopidos $C$. cincta y $C$. asoralis mostraron una mayor sensibilidad, pues el extracto acuoso al $10 \%$ a $48 \mathrm{~h}$ de exposición produjeron $75 \%$ y $20.1 \%$ de mortalidad larvaria, respectivamente. El molle acuoso produjo efectos ovicidas sobre estos dos neuropteros; sin embargo, en C. externa no se observaron efectos en la eclosión de los huevos (Iannacone \& Lamas 2003a).

En adultos de Trichogramma pintoi Voegelé se presentó $28.9 \%$ de mortalidad al molle acuoso al 10\% (Iannacone \& Lamas 2003a). En el presente estudio, $O$. insidiosus al $10 \%$ de molle acuoso produjo efectos significativos de $41.1 \%$ de mortalidad. En cambio, el adulto de T. remus no presentó efectos significativos al molle acuoso al 10\%. De igual forma el parasitoide Copidosoma koehleri Blanchard no presentó efecto significativo alguno bajo la acción del molle acuoso (Iannacone \& Lamas 2003a).

Ruffinengo et al. (2005) en un análisis comparativo entre nueve plantas con propiedades acaricidas-insecticidas, ha notado que $S$. molle tiene el mayor indice de selectividad (IS > 16) y un menor riesgo de efecto adverso sobre la abeja $A$. mellifera.

La aplicación de estos índices de selectividad (IS) en los binomios: Phthorimaea operculella (Zeller)/ C. externa (IS $=8.1)$, P. operculella/ T. pintoi (IS = 3.16) y P. operculella/ C. koehleri (IS =1.1), muestran la ventaja del uso del extrato acuoso de molle al 10\%, y su menor efecto en los organismos benéficos, pues en los dos primeros casos dieron valores > a 2 (Ruffinengo et al. 2005). Sin embargo, estos mismos extractos acuosos al 10\% mostraron efectos adversos 


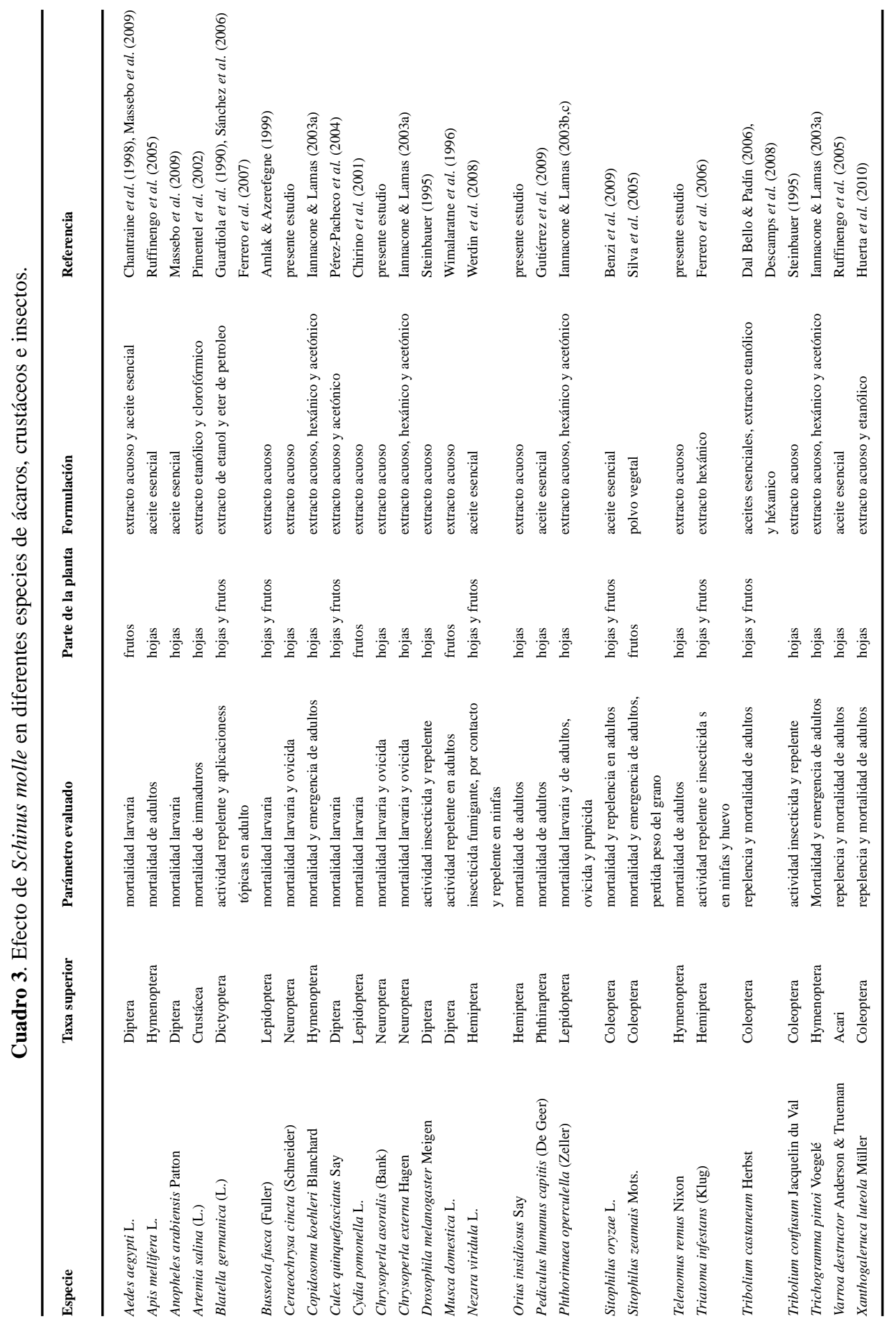


principalmente en $C$. cincta y $O$. insidiosus (Cuadros 1 y 2). Por lo que el uso simultáneo del extracto de molle con estos dos controladores biológicos no sería compatible dentro de un programa integrado de plagas. En todo caso, el empleo de un controlador biológico y de un extracto botánico podría ser alternado en el manejo integrado de plagas. De esta forma se estaría favoreciendo el uso de alternativas agroecológicas sustentables.

Según la clasificación de la IOBC/WPRS (Sterk et al. 1999) el extracto acuoso de molle al $20 \%$ es inocuo (mortalidad < 50\%) para T. remus y C. asoralis. En cambio, ligeramente nocivo (50 y $79 \%$ de mortalidad) para $O$. insidiosus, y nocivo (> 90\% de mortalidad) para C. cincta.

La mayor sensibilidad larvaria por contacto y el mayor efecto en la eclosión de huevos por inmersión de $C$. cincta por acción del extracto acuoso de $S$. molle en comparación a las otras tres especies de enemigos naturales, y principalmente con $C$. asoralis no puede ser bien explicada con los resultados del presente trabajo. Posiblemente los mecanismos de detoxificación al molle sean ligeramente diferentes en comparación a los otros tres controladores biológicos. Un patrón opuesto ha sido observado para Ceraeochrysa cubana (Hagen, 1861), que resultó ser más tolerante a la azadirachtina y al bifentrina que Chrysoperla rufilabris (Burmeister, 1839) a nivel de huevos, larvas y adultos (Schuster \& Stansly 2000).

Finalmente, se recomienda realizar ensayos de campo en cultivos como algodón y espárrago con extractos acuosos de $S$. molle sobre ciertas plagas donde ha observado efectividad, para obtener información de su eficacia insecticida y su empleo en conjunto con controladores biológicos en el manejo integrado de plagas.

\section{LITERATURA CITADA}

Abbott, W.S. 1925. A method of computing the effectiveness of an insecticide. Journal of Economic Entomology, 18: 265-267.

Amlak, G. \& F. Azerefegne. 1999. Insecticidal activity of chinaberry, endod and pepper tree against the maize stalk borer (Lepidoptera: Noctuidae) in Southern Ethiopia. International Journal of Pest Management, 45: 9-13.

Benzi, V., Stefanazzi, N. \& A.F. Ferrero. 2009. Biological activity of essential oils from leaves and fruits of pepper tree (Schinus molle L.) to control rice weevil (Sitophilus oryzae L.). Chilean Journal of Agricultural Research, 69: 154-159.

Bortoli, S. A., A.T. De Murata, R.S. Narciso \& C.H. De Brito. 2005. The nutritional aspects of Ceraeochrysa cincta Schneider, 1851 (Neuroptera: Chrysopidae) and different preys. Revista de Agricultura Piracicaba, 80: 1-11.

Bueno, R. C. O., T.R. Carneiro, D. Pratissoli, A.F. Bueno \& O.A. Fernandes. 2008. Biology and termal requirement of Telenomus remus reared on fall armyworm Spodoptera frugiperda eggs. Ciência Rural, 38: 1-6.

Calow, P. 1993. Handbook of ecotoxicology. Blackwell Science Ltd. Sheffield, UK. Vol. I., 478 pp.

Cano, V.E. 2001. Cría masiva de Trichogramma pretiosum, Sitotroga cerealella y Chrysoperla externa. Manejo Integrado de Plagas (Costa Rica), 60: 93-96. 
Cardoso, E. R., S. De Freitas, H.T. Nunes \& I.G.A. Pessoa. 2004. Seletividade de fungos entomopatogénicos para larvas de primeiro instar de Ceraeochrysa cincta (Neuroptera: Chrysopidae) utilizando torre de Potter. Arquivos do Instituto Biológico, 71: 129-130.

Carvalho, G.A., F.A. Drummond, J.L.R. Ulhoa \& L.C.D. Rocha. 2002. Effect of insecticides on Orius insidiosus (Say, 1832) (Hemiptera: Anthocoridae). Ciência e agrotecnologia, Lavras, 26: 5256.

Cave, R.D. 2000. Biology, ecology and use in pest management of Telenomus remus. Biocontrol, 21: 21-26.

Chantraine, J.M., D. Laurent, C. Ballivian, G. Saavedra, R.L. Ibañez \& A. Vilaseca. 1998. Insecticidal activity of essential oils on Aedes aegypti larvae. Phytotherapy Research, 12: 350-354.

Chirino, M., M. Cariac \& A.A. Ferrero. 2001. Acticidad insecticida de extractos crudos de drupas de Schinus molle L. (Anacardiaceae) sobre larvas neonatas de Cydia pomonella L. (Lepidoptera: Tortricidae). Boletín de Sanidad Vegetal Plagas, 27: 305-314.

CONABIO 2009. En www.conabio.gob.mx/conocimiento/info_especies/arboles/doctos/3-anaca4m.pdf - leído el 07 de enero del 2009.

Dal Bello, G. \& S. Padín. 2006. Olfatómetro simple para evaluar la actividad biológicia de aleloquímicos vegetales en Tribolium castaneum Herbst (Coleoptera: Tenebrionidae). Agrociencia, 10: 2326.

Descamps, L.R., N. Stefanazzi, C. Sánchez-Chopa \& A.A. Ferrero. 2008. Actividad biológica de extractos vegetales de Schinus molle var. areira (Anacardiaceae) en Tribolium castaneum Herbst. (Insecta, Coleoptera, Tenebrionidae), plaga de grano almacenado. Boletín de Sanidad Vegetal. Plagas, 34: 595-606.

Dikshit, A., A. A. Naqvi \& A. Husain. 1986. Schinus molle: a new source of natural fungitoxicant. Applied and Environmental Microbiology, 51: 1085-1088.

Ferrero, A.A., J.O.W. González \& C.C. Sánchez. 2006. Biological activity of Schinus molle on Triatoma infestans. Fitoterapia, 7: 381-383.

Ferrero, A.A., C.C. Sánchez, J.O.W. González \& R.A. Alzogaray. 2007. Repellence and toxicity of Schinus molle extracts on Blattella germanica. Fitoterapia, 78: 311-314.

Gazit, Y., W.J. Lewis \& J.H. Tumlinson. 2002. Arrestment of Telenomus remus (Hymenoptera: Scelionidae) by a kairomone associated with eggs of its host, Spodoptera frugiperda (Lepidoptera: Noctuidae). Biological Control, 6: 283-290.

Gonzáles, O.E.V. \& C. Reguillón. 2002. A new species of Chrysoperla (Neuroptera: Chrysopidae) from Argentina. Revista de la Sociedad Entomologica Argentina, 61: 47-50.

Guardiola, V.G, P. De Miguel \& E. Primo. 1990. Repellent activity against Blattella germanica of components of Schinus molle L. Revista de Agroquímicos y Tecnología de Alimentos, 30: 341-346.

Gutierrez, M.M., N. Stefanazzi, J.G. Werdin, V. Benzi \& A.A. Ferrero. 2009. Actividad fumigante de aceites esenciales de Schinus molle (Anacardiaceae) y Tagetes terniflora (Asteraceae) sobre adultos de Pediculus humanus capitis (Insecta; Anoplura; Pediculidae). Boletín Latinoamericano y del Caribe de Plantas Medicinales y Aromaticas, 8: 176-179.

Hernández-Albiter, R.C., N.L.L. Barrera, B.S. Bautista \& L.L. Bravo. 2007. Antifungal potencial of crude extracts on conidial germination of two isolates of Colletotrichum gloeosporioides (Penz.) Penz. And Sacc. Revista Mexicana de Fitopatología, 25: 180-185.

Huerta, A., I. Chiffelle, K. Puga, F. Azúa \& J.E. Araya. 2010. Toxicity and repellence of aqueous and ethanolic extracts from Schinus molle on elm leaf beetle Xanthogaleruca luteola. Crop Protection, 29: $1118-1123$.

Iannacone, J. \& L. Alvariño. 2005. Selectividad del insecticida cartap empleando bioensayos con organismos no destinatarios. Ecología Aplicada, 4: 91-104. 
Iannacone, J. \& G. Lamas. 2002. Efecto de dos extractos botánicos y de un insecticida convencional sobre el depredador Chrysoperla externa. Manejo Integrado de Plagas y Agroecología (Costa Rica), 65: 92-101.

Iannacone, J. \& G. Lamas. 2003a. Efectos toxicológicos de extractos de molle (Schinus molle) y lantana (Lantana camara) sobre Chrysoperla externa (Neuroptera: Chrysopidae), Trichogramma pintoi (Hymenoptera: Trichogrammatidae) y Copidosoma koehleri (Hymenoptera: Encyrtidae). Agricultura Técnica (Chile), 63: 347-360.

Iannacone, J. \& G. Lamas. 2003b. Efecto insecticida de cuatro extractos botánicos y del cartap sobre la polilla de la papa Phthorimaea operculella (Zeller) (Lepidoptera: Gelechiidae), en el Perú. Entomotropica, 18: 95-105.

Iannacone, J. \& G. Lamas. 2003c. Plantas biocidas usadas para el control de la polilla de la papa, Phthorimaea operculella (Zeller) (Lepidoptera: Gelechiidae). Revista peruana de Entomología, 43: 79-87.

Iannacone, J., L. Alvariño, C. Caballero \& J. Sánchez. 2000. Cuatro ensayos ecotoxicológicos para evaluar lindano y clorpirifos. Gayana, 64: 139-146.

Iannacone, J., L. Alvariño, Y. Murrugarra, A. Arrascue, M. Alayo \& N. Salazar. 2008. Selectividad del insecticida metamidofos en ocho organismos terrestres no destinatarios. Journal of Brazilian Society of Ecotoxicology, 3: 23-34.

López-Arroyo J. A., C.A. Tauber \& M.J. Tauber. 1999a. Intermittent oviposition and remating in Ceraeochrysa cincta (Neuroptera: Chrysopidae). Annals of the Entomological Society of America, 92: 587-593.

López-Arroyo J. A., C.A. Tauber \& M.J. Tauber. 1999b. Comparative life histories of the predators Ceraeochrysa cincta, C. cubana, and C. smithi (Neuroptera: Chrysopidae). Annals of the Entomological Society of America, 92: 208-217.

Ludwig, S. \& R. Oetting. 2001. Effect of spinosad on Orius insidiosus (Hemiptera: Anthocoridae) when used for Frankliniella occidentalis (Thysanoptera: Thripidae) control on greenhouse pot chrysanthemums. Florida Entomologist, 84: 311-313.

Massebo, F., M. Tadesse, T. Bekele, M. Balkew \& T. Gebre-Michael. 2009. Evaluation on larvicidal effects of essential oils of some local plants against Anopheles arabiensis Patton and Aedes aegypti Linnaeus (Diptera, Culicidae) in Ethiopia. African Journal of Biotechnology, 8: 4183-4188.

Morales, J.S., V.J.S. Gallardo, C. Vásquez \& Y. Ríos. 2000. Patrón de emergencia, longevidad, parasitismo y proporción sexual de Telenomus remus (Hymenoptera: Scelionidae) con relación al cogollero del maíz. Bioagro, 12: 47-54.

Morales, J.S., V.J.S. Gallardo, C. Vásquez \& Y. Ríos. 2001. Respuesta funcional de Telenomus remus (Hymenoptera: Scelionidae) a los huevos de Spodoptera frugiperda (Lepidoptera: Noctuidae). Bioagro, 13: 49-55.

Murthy, K.S., N.S. Rao, R.J. Rabinfra \& S.K. Jalali. 2004. Age related parasitisation potential of the eggs parasitoid Telenomus remus (Scelionidae: Hymenoptera) on certain lepidopterous hosts. Journal of Entomology Research, 28: 33-36.

Nuñez, Z.E. 1988. Ciclo biológico y crianza de Chrysoperla externa y Ceraeochrysa cincta (Neuroptera: Chrysopidae). Revista Peruana de Entomología, 31: 76-82.

Pérez-Pacheco, R., H.C. Rodriguez, R.J. Lara, B.R. Montes \& V.G. Ramirez. 2004. Toxicidad de aceites, esencias y extractos vegetales en larvas de mosquito Culex quinquefasciatus Say (Diptera: Culicidae). Acta Zoologica Mexicana (n.s.), 20: 141-152.

Pimentel, M.A.B., M.G. Pizzolatti \& I.M.B. Costa. 2002. An application of the brine shrimp bioassay for general screening for Brazilian Medicinal plants. Acta Farmacológica Bonaerense, 21: 175-178. 
Ramírez, D.M., A.I. López, H.A. González \& Z.M.H. Badii. 2007, Rasgos biológicos y poblacionales del depredador Cereaeochrysa sp. nr. cincta (México) (Neuroptera: Chrysopidae). Acta Zoologica Mexicana (n.s.), 23: 79-95.

Ruffinengo, S., M. Eguaras, I. Floris, C. Faverin, P. Bailac \& M. Ponzi. 2005. LD 50 and repellent effects of essential oils from Argentinian wild plant species on Varroa destructor. Journal of Economic Entomology, 98: 651-655.

Sánchez C. C., R. Alzogaray \& A. Ferrero. 2006. Repellency assays with Schinus molle var. areira (L.)(Anacardiaceae) essential oils against Blattella germanica L. (Blattodea: Blattellidae). BioAssay, 1(6): 1-3.

Schuster, D.J. \& P.A. Stansly. 2000. Response of two lacewing species to biorational and broad-spectrum insecticide. Phytoparasitica, 28: 297-304.

Silva, G., O. Orrego, R. Hepp \& M. Tapia. 2005. Búsqueda de plantas con propiedades inecticidas para el control de Sitophilus zeamais em maíz almacenado. Pesquisas Agropecuarias Brasileiras, 40: 11-17.

Steinbauer, M.J. 1995. The insecticidal and repellent activity of Schinus molle L. (Anacardiaceae) against Drosophila melanogaster Meigen (Diptera: Drosophilidae) and Tribolium confusum Jacquelin du Val (Coleoptera: Tenebrionidae). General and applied Entomology, 26:13-18.

Sterk, G., S.A. Hassan, M. Baillod, F. Bakker, F. Bigler, S. Blümel, H. Bogenschütz, E. Boller, B. Bromand, J. Brun, J.N.M. Calis, J. Coremans-Pelseneer, C. Duso, A. Garido, A. Grove, U. Heimbach, H. Hokkanen, J. Jacas, L. Lewis, L. Moreth, L. Polgar, L. Rovesti, L. SamsoePetersen, B. Sauphanor, L. Schaub, A. Stäubli, J.J. Tuset, M. Vainio, M. Van De Veire, G. Viggiani, E. Viñuela, \& H. Vogt. 1999: Results of the seventh joint pesticide testing programme carried out by th IOBC/WPRS working group "Pesticides and Beneficial Organisms". Biocontrol, 44: $99-117$.

Studebaker, G.L. \& T.J. Kring. 2003. Effects of insecticides on Orius insidiosus (Hemiptera: Anthocoridae), measured by field, greenhouse and Petri dish bioassays. Florida Entomologist, 86:178185.

Tauber, C.A. \& C.A.T. De León. 2001. Systematics of green lacewing (Neuroptera: Chrysopidae): larvae of Ceraeochrysa from Mexico. Annals of the Entomological Society of America, 94: 197-209.

Throne, J.E., D.K. Weaver, V. Chew \& J.E. Baker. 1995. Probit analysis of correlated data: multiple observations over time at one concentration. Journal of Economic Entomology, 88: 1510-1512.

Waddill, V.H. 1978. Contact toxicity of four synthetic pyrethroids and methomyl to some adult insect parasites. Florida Entomologist, 61: 27-30.

Weber, C. 1993. Methods for measuring the acute toxicity of effluents and receiving waters to freshwater and marine organisms. EPA/600/4-90/027F.

Werdin, J.O., A.P. Murray \& A.A. Ferrero. 2008. Bioactividad de aceites esenciales de Schinus molle var. areira (Anacardiaceae) en ninfas II de Nezara viridula (Hemiptera: Pentatomidae). Boletín de Sanidad Vegetal Plagas, 34: 367-376.

Wimalaratne P. D. C., K.N. Slessor, J.H. Borden, L.J. Chong. \& T. Abate. 1996. Isolation and identification of house fly, Musca domestica L., repellents from pepper tree, Schinus molle L. Journal of Chemical Ecology, 22: 49-59.

Wu, G., S. Jiang \& T. Miyata. 2004a. Seasonal changes of metamidophos susceptibility and biochemical properties in Plutella xylostella (Lepidoptera: Yponomeutidae) and its parasitoid Cotesia plutella (Hymenoptera: Braconidae). Journal of Economic Entomology, 97: 1689-1698.

Wu, G., S. Jiang \& T. Miyata. 2004b. Effects of sinergists on toxicity of six insecticides in parasitoid Diaretiella rapae (Hymenoptera: Aphidiidae). Journal of Economic Entomology, 97: 2057-2066. 